\title{
Diseño y propuesta de un modelo de análisis para la gestión de la comunicación corporativa local
}

\section{Design proposal of an analysis model for the management of local corporate communication}

\author{
Dr. Óscar Mesa Medina \\ omesamedina@gmail.com \\ ORCID: https://orcid.org/0000-0002-1804-9705 \\ Dr. Rafael Marfil Carmona \\ Universidad de Granada \\ rmarfil@ugr.es \\ ORCID: https://orcid.org/0000-0001-9688-7088
}

Universidad de Gales / Escuela Superior de Comunicación y Marketing de Granada

Resumen: La comunicación política, ya sea organizada o personal, tiene una gran relevancia en la actualidad. El sector profesional de las relaciones públicas en corporaciones locales se va adaptando progresivamente a una interacción dinámica y cercana a la ciudadanía, propia de una cultura digital que ha roto el marco tradicional. A diferente escala, la comunicación política de los municipios resulta clave para que estos se hagan visibles en la aldea global, promocionando sus actividades y decisiones de gestión. Este trabajo propone un modelo de análisis comparativo para estudiar la comunicación política de pequeños ayuntamientos, basado en criterios sociodemográficos, económicos y comunicacionales. Se trata de parámetros abarcables que ofrecen una visión general, estableciendo la relación entre tamaño, capacidad de los municipios y su apuesta por la comunicación institucional. La metodología de estudio diseñada abarca, por un lado, parámetros cuantitativos relacionados con la capacidad económica. Por otro lado, se propone el estudio de sus gabinetes de prensa y de las herramientas comunicativas.

Se ofrecen los resultados de una puesta en prueba tras analizar cinco ayuntamientos del Área Metropolitana de Granada (España), de los que se desprende un balance positivo desde el punto de vista profesional de la gestión de su comunicación institucional. La herramienta ha resultado útil para analizar, en un espacio breve de tiempo, el interés y la capacidad de comunicar que tienen las cinco corporaciones locales. En este sentido, 
se ha detectado poca adaptación a las nuevas formas de comunicación política e institucional en comparación con el mundo empresarial.

Abstract: We are witnessing the era that gives the greatest power to political communication, whether it be organized or personal. The professional public relations sector in local authorities is progressively adapting to citizens, with a dynamic interaction with them. Digital culture has broken the traditional framework of relations. In this sense, the political communication of the municipal local governments is key for them to become visible in the global village, promoting their activities and management decisions. This paper proposes a comparative analysis model to study the political communication of small-town councils, based on sociodemographic, economic and communicational criteria. These are comprehensive parameters that offer a global vision, establishing the relationship between size, capacity of the municipalities and their institutional communication. The methodology designed covers, on the one hand, quantitative parameters related to economic capacity. On the other hand, it is proposed to study its press offices and communication tools. In the results, we report on a test carried out after analysing five Town Council in the Metropolitan Area of Granada (Spain). The balance is positive from the professional point of view of the management of your institutional communication. The tool has proved useful in analysing, in a short time, the interest and ability of the five municipalities to communicate. Finally, there is poor adaptation to new forms of political and institutional communication compared to the business world.

Palabras clave: Periodismo, Comunicación Institucional, Ayuntamientos, Gabinetes de Prensa, Relaciones Públicas.

Keywords: Journalist, Institutional Communication, Town Council, Press Office, Public Relations.

\section{INTRODUCCIÓN}

Asistimos a una época que confiere una gran relevancia a la comunicación política, en lo que se puede denominar una verdadera "batalla por la opinión pública" (Castells, 2008), ya sea organizada o resultante del liderazgo personal. La política tradicional ha ido aceptando una interacción más dinámica y cercana al ciudadano, propia de la cultura digital (Muraro, 1991). La irrupción de fenómenos políticos como Donald Trump en Estados Unidos o el partido Podemos en España se explican, en buena medida, por una comunicación que ha roto el marco tradicional (Rodríguez-Andrés, 2016).
En el ámbito institucional, la comunicación política de los municipios resulta clave para hacerse visibles, promocionando sus actividades y decisiones de gestión a públicos externos e internos, pues son las nuevas tecnologías las que delimitan si existes o no existes en la Aldea Global (Thompson, 2015). De esta forma, dichas instituciones obtendrán un buen posicionamiento en parámetros clave como notoriedad y actitud (Domínguez, 2009). Estamos en el terreno de la comunicación de intangibles, vinculados a la imagen institucional, así como del esfuerzo en cada acción por lo que se puede denominar "reputación corporativa", entendida como lo relacionado con el comportamiento institucional, la seriedad en la gestión 
y la valoración global de acciones (Villafañe, 2004, p. 28).

Todas las localidades tienen elementos que difundir, pero no todas son conocidas. ¿La clave? La comunicación, siempre. En la mayor parte de los contextos locales no existe el apoyo de los denominados Think Tanks, organizaciones independientes que llevan a cabo la difusión de sus investigaciones e ideas sobre asuntos de política pública, realizando aportaciones para la credibilidad del sector (Castillo-Esparcia y Smolak-Lozano, 2016, p. 329). Por su parte, desde una perspectiva mucho más cercana a la ciudadanía, los ayuntamientos, en su quehacer diario, tienen que dar respuesta a problemáticas muy concretas por sí mismos.

En ese día a día, las corporaciones locales comparten la necesidad de posicionarse y, en mayor o menor medida, cuentan con los recursos para ello. Actualmente no es necesario invertir grandes cantidades de dinero en publicidad. De hecho, se trata de una opción desaconsejable. Son más eficaces otras alternativas, como la generación de puclicity, es decir, la generación de impactos informativos sin pago de espacios comerciales, lo que requiere una buena gestión informativa. La inversión pasa, sobre todo, por profesionalizar el departamento de comunicación con, al menos, una persona responsable y bien formada para ello, así como dotar a ese departamento con una partida presupuestaria básica, tal y como afirma Joan Costa (2014).

De este modo, se trabaja erróneamente con la idea preestablecida en la cultura política española de que la comunicación puede llevarla a cabo cualquiera, especialmente en la política a pequeña escala (Costa, 2015). Lo que sí es indudable es el potencial de la comunicación local como vía de vertebración social, como afirma Lluís Costa:

La vitalidad de la comunicación de proximidad
tiene una relación directa con la vitalidad
asociativa de un territorio, que cuanto más activo
es, social y políticamente, más necesidad tiene
de comunicarse. La comunicación local, pues,
es un elemento importante de vertebración de la
sociedad local. (2015, p.13) Algunas grandes ciudades españolas ejemplifican esta transformación de su comunicación. Hoy día se está trabajando en esta línea desde los ayuntamientos grandes, con un buen espejo donde mirarse en Bilbao, que ha pasado de sombría ciudad industrial a lujoso destino turístico (Álvarez, 1999). También ha sucedido durante la última década en Barcelona, con un proyecto para promocionar el Barrio Gótico (Cócola, 2010). Es fundamental tener presente los valores del patrimonio intangible de cada localidad: “... Se propone posicionarse identitariamente $y$ transmitir, si cabe, un espíritu vivencial, concretado en una marca de territorio, en lo que representa la transmisión de determinados valores, exaltando el patrimonio intangible implícito en la ciudad" (Jiménez y San Eugenio, 2009, pp. 286-287). Para ello, resulta absolutamente necesario contar con una estrategia online y un trabajo en redes sociales que permita llegar a las generaciones más jóvenes y transmitir una imagen globalizada (Ostio, 2012). En este sentido, la comunicación tiene que optar por campañas promocionales de $360^{\circ}$ - combinando elementos on y off line -, que deben ser coherentes con la estrategia global de comunicación de la institución.

Las ciudades se han convertido en sujetos de consumo, en marcas con identidades y valores propios (Sáez, 2011), por lo que el desarrollo y potenciación de una buena imagen de marca, como ocurre con 
las empresas y organizaciones, será fundamental para competir en el mercado turístico y de inversión. Las ciudades, y por ende todos los municipios, deben ser conscientes de sus valores, cultura, personalidad, atributos y público objetivo al que se dirigen. Cualquier localidad puede crear una imagen de marca y posicionar aquellos factores que la hacen diferente, como podemos observar en el caso de Jun (Granada, España) con su posicionamiento en nuevas tecnologías y su uso en la gestión municipal. La clave, quizás, sea comunicar para un público específico y definido, ser eficientes a la hora de llegar hasta él. ¿Cómo lograrlo? Profesionalizando el departamento y siendo capaces de adaptarse a lo que, realmente, supone un cambio de paradigma debido a los nuevos medios digitales, que se basan en un continuo proceso de interacciones:

\begin{abstract}
A este respecto, uno de los principales problemas que se suscitan a la hora de afrontar el cambio de paradigma comunicacional en el escenario digital es que se siguen planteando las mismas estrategias de comunicación externa convencional (notas de prensa, ruedas de prensa, comunicados escritos), sin comprender que las plataformas digitales no son medios unidireccionales y que el concepto de "comunidad digital" exige interacción comunicativa entre emisor/es y receptor/es, pero además, que el contenido compartido en un canal institucional no será la única información que emerge en la unidad, sino que es la comunidad con sus interacciones lo que le otorga valor informativo. (Romero-Rodríguez y Mancinas-Chávez, 2016, p. 117)
\end{abstract}

Desde este punto de vista, hay que tener presente que, superando el mensaje unidireccional y la pretensión del control absoluto de la agenda setting, la comunicación corporativa contemporánea debe valorar la importancia de lo que Carmen Marta-Lazo y José Antonio Gabelas denominan "factor relacional" en la comunicación digital (2016), una perspectiva que, apoyándose en el elemento humanizador que necesita este ámbito, es sin duda el "núcleo del paradigma de las relaciones públicas de vanguardia" (Pulido Polo, 2013, p. 145).

De esta forma, si para el sector empresarial "los mercados son conversaciones" (Levine, Locke, Sears y Winbrger, 2008), las corporaciones deben aplicar esta filosofía de trabajo en lo que podría denominarse como "Relaciones Públicas", consideradas como una “... actividad que establece procesos de adaptación intra y extra con los públicos de la organización de una manera recíproca", estableciendo los cambios necesarios para las relaciones mutuas (CastilloEsparcia, 2010, pp. 61-62), si bien se puede hacer referencia directamente a "Relaciones Públicas 2.0" (Aced, 2013), ya que hoy día es imprescindible el aprovechamiento de los medios digitales. Se trata, qué duda cabe, de "mercados" de información y notoriedad.

En este sentido, conscientes de la profunda transformación que supone ese entorno digital e interactivo de "hipermediaciones" (Scolari, 2008), en el presente trabajo se propone una herramienta para analizar el modelo de gestión en la comunicación de instituciones públicas locales, una primera aproximación que ha sido puesta en prueba con 5 municipios de la provincia de Granada (España). En la línea de algunas investigaciones y trabajos académicos centrados en la profesionalización de la comunicación corporativa (Armendáriz, 2015; Capriotti, 2016; Escobar y Gómez, 2015; Martín, 2007; Subiela Hernández, 2013; Villafañe, 2011), se ha tenido en cuenta también cuáles son los rasgos específicos de la gestión de la comunicación local (Vázquez Gestal y Fernández Souto, 2014), así como 
aquellas características especiales de las instituciones públicas y de las corporaciones municipales (García Santamaría y Martín Matallana, 2017; Sureda Pons y Torres Plana, 2016). Igualmente, es imprescindible valorar el proceso de adaptación de estas entidades a la cultura digital (Almansa Martínez, 2008; Campillo Alhama y Ramos-Soler, 2013; Catalina García y García Jiménez, 2013; Sabés Turmo, 2008). Partiendo de esas investigaciones previas, se propone en este trabajo un modelo de análisis de la comunicación corporativa local.

Considerando el contexto de profundos cambios que van de lo más próximo a lo global, se propone una herramienta que permita visualizar sencilla y esquemáticamente las cualidades necesarias para comunicar en los ayuntamientos, así como la realización de un posterior análisis comparativo de la comunicación política que llevan a cabo. Con el fin de realizar un test previo de este instrumento, se han elegido cinco municipios del Área Metropolitana de Granada. Este análisis, adaptable a un estudio personalizado de modelos de gestión, atiende a criterios sociodemográficos y comunicativos para dar una visión lo más completa posible de un objeto de estudio en permanente transformación.

\section{OBJETIVOS}

En cuanto a los objetivos del presente estudio, se tienen en cuenta tres vías de trabajo complementarias:

1. Diseñar y proponer un modelo de análisis de la comunicación corporativa local, aplicable a los gabinetes de prensa de los ayuntamientos.
2. Poner en prueba la aplicación del modelo diseñado para una muestra pequeña de municipios.

3. Destacar la importancia de la comunicación corporativa local y estudiar si se ha apostado o no por la profesionalización en ayuntamientos que no son capital de provincia.

\section{METOdOLOGÍA}

Esta investigación se ha basado en una metodología cuantitativo-cualitativa, considerándola la forma más completa para trabajar con un objeto de investigación social, si bien la diferencia entre lo cualitativo y cuantitativoes cadavez más difusa (Igartuay Humanes, 2010, p. 75), siendo habitual su integración, sobre todo en el marco investigador de Ciencias Sociales. Los datos numéricos permiten una comparación clara a la hora de establecer rangos y eficacias, así como a la hora de ordenar posteriormente los ayuntamientos según su posicionamiento en determinados parámetros. Por su parte, el factor cualitativo permite evaluar aspectos que, si bien podrían ser cuantificables, se puede clarificar y profundizar más cuando se da prioridad a su componente interpretativo, ya que éste se acerca mejor a la realidad del objeto, aportando matices imposibles de obtener mediante números. En este sentido, es importante la confianza en el criterio analítico de la persona o el equipo de investigación.

La propuesta se basa, por tanto, en un sistema de investigación deductivo, vinculando una serie de teorías previas respecto de la comunicación corporativa local para aplicarlas a la secuencia de trabajo de un modelo de análisis para el estudio de una muestra concreta. Se intenta implementar, así, una transferencia desde la teoría a la realidad práctica. Se parte de la base 
de la progresiva mejora de estos departamentos, ya que "... las organizaciones saben que tienen que cubrir sus necesidades comunicativas" (Almansa Martínez, 2004, p. 14). En respuesta a esa necesidad, se aporta en este texto una metodología que permita estudiar los gabinetes en sus rasgos generales, en su funcionamiento y arquitectura básica.

El propósito de la herramienta diseñada, así como de la puesta en prueba realizada, tiene un claro fin exploratorio, en el que se pretende realizar una aproximación inicial a una nueva realidad, absolutamente emergente, como es la transformación de la comunicación en las corporaciones locales. Un aspecto de gran impacto social, ya que llega de forma directa a gran parte de la ciudadanía, residente en municipios medianos o pequeños. La necesidad de establecer criterios para organizar una información compleja le confiere ese carácter exploratorio, pero también descriptivo con respecto a las localidades en las que se ha puesto en práctica la herramienta de análisis diseñada. En ese objetivo descriptivo hay una incidencia especial en el detalle y la justificación de qué aspectos deben medirse. El carácter explicativo en una mayor profundidad, así como la comparación de variables, que abarca solo lo esencial y básico en este trabajo, quedaría para la sugerencia de futuras investigaciones que consideren analizar una muestra más amplia y representativa.

Se propone un modelo de análisis comparativo, operativo para el diagnóstico y relativamente sencillo de aplicar, en el que se consideren a un mismo tiempo criterios sociodemográficos, económicos y comunicativos. Se trata de parámetros abarcables que ofrecen una visión global del objeto, estableciendo la relación entre el tamaño y capacidad de los municipios, siempre desde la valoración de su apuesta por la comunicación institucional. Para ello, se ha diseñado una metodología de estudio basada en el cuestionario como herramienta para ofrecer una visión global. Se abarcan, por un lado, parámetros cuantitativos que inciden en la capacidad económica: tamaño, renta, población, presupuesto y partida destinada a comunicación. Por otro lado, se propone el estudio cualitativo de sus gabinetes de prensa: existencia, personal implicado y su formación, horario y funciones del puesto de trabajo, herramientas que se utilizan o el conocimiento que tienen sobre sus valores diferenciales aquellos que deben comunicarlos, entre otros aspectos destacados.

La definición de variables y la aportación de esa ficha de análisis es un trabajo de elaboración propia, que debe considerarse como una propuesta de partida, resultante de la revisión teórica y la valoración de las investigaciones a las que se ha hecho referencia en la introducción. Su estructura se fundamenta en la consideración básica de los datos externos sociodemográficos del municipio, así como internos, relativos al presupuesto municipal y a las decisiones de gestión en lo que respecta a las funciones de comunicación. Se opta, en este sentido, por la difusión en el ámbito científico de esta herramienta como vía para solicitar y aplicar mejoras. La necesidad de establecer unos criterios mínimos, aplicables a multitud de contextos con una gran diversidad, hacen conveniente que la herramienta diseñada solo se centre en los criterios elementales. Su desarrollo y aplicación debe revertir en una progresiva mejora, tanto en el detalle como en la capacidad de adaptabilidad.

Además, como validación de esta herramienta de análisis resultaba necesario aplicarla a un estudio de 
municipios reales. En la puesta en prueba realizada se ha seleccionado una muestra de 5 municipios del Área Metropolitana de Granada: Alfacar, Albolote, Cúllar Vega, Maracena y Monachil. La elección de esta muestra concreta responde a su cercanía física y accesibilidad por parte de los investigadores, con la ventaja de estar circunscritos dentro de un área administrativa concreta y conocida. Por otro lado, aportan diversidad en tamaño y condición. De esta forma se ha obtenido finalmente una herramienta que pretende ser completa, útil y sencilla de utilizar. Su difusión, así como los primeros resultados, pretenden fomentar un proceso de mejora e intercambio de perspectivas por parte de la comunidad investigadora en Comunicación.

\section{RESULTADOS}

\subsection{Propuesta de modelo de análisis}

La herramienta propuesta para el análisis comparativo de la comunicación política que llevan a cabo los municipios, y que incide ineludiblemente en la imagen de marca y reputación, puede dividirse en dos partes:

- Análisis de los recursos que invierte cada ayuntamiento en comunicar, tanto de carácter absoluto como relativo a sus posibilidades. Se trata de datos puramente cuantitativos.

- Análisis de los gabinetes de prensa o de las iniciativas o soluciones de gestión que los sustituyen. Esta línea de trabajo se basa en la valoración cualitativa del criterio municipal y su estrategia de trabajo en comunicación.
Para analizar la capacidad económica y los recursos invertidos por cada municipio, se ha recabado la siguiente información:

\section{Datos demográficos y sociológicos}

- Población. Un valor que normalmente incide de forma directa en el presupuesto del municipio.

- Tamaño. La extensión en kilómetros cuadrados, que también debería tener influencia en el presupuesto en materia impositiva.

\section{Datos económicos}

- Renta per cápita. Puede influir en la propia necesidad de comunicar y en la orientación de las acciones a realizar, ofreciendo información sobre el perfil medio y las necesidades de la ciudadanía.

- Presupuesto anual del municipio. De él derivará la posibilidad de invertir más o menos en comunicación.

- Partida presupuestaria destinada a comunicación. La cantidad que se destina de forma directa a comunicar, como puede ser el montante global correspondiente a salarios, campañas publicitarias o mantenimiento de la web o de un medio de comunicación propio. Aunque la tendencia en marketing y publicidad es buscar vías alternativas a la publicidad tradicional, un presupuesto elevado sí que evidencia las intenciones y el criterio seguido. Es importante analizar también la planificación y capacidad de previsión en las inversiones cuando sea posible. 
A la hora de analizar las opciones municipales en materia de comunicación en lo que concierne a su gabinete de prensa, se han analizado las siguientes cuestiones:

- Existencia del propio gabinete. Es la clave del estudio, pues esclarece el interés del ayuntamiento en profesionalizar su comunicación. Su ausencia también puede deberse a incapacidad económica para afrontarlo, pero no a ser un municipio de escaso interés, por ejemplo.

- Quién realiza las funciones de comunicar en caso de no existir gabinete. Puede que nadie tome las riendas de la comunicación o puede que algún miembro del consistorio incluya esas funciones en su tarea diaria. Lo importante es su funcionalidad.

- Existencia de un horario cerrado y tiempo específico dedicado. Es importante que la persona encargada de comunicar esté disponible un número de horas semanales y que pueda ser localizada en su horario de trabajo. De esta forma, cualquier consulta o propuesta pasará por sus manos con fluidez.

- Si el responsable es profesional de la comunicación. Cualquiera puede comunicar, pero no cualquiera puede comunicar bien. No es indispensable legalmente ser titulado en Ciencias de la Comunicación - o equivalente legal -, aunque sería lo deseable, pero sí es recomendable demostrar un conocimiento teórico que pueda ser llevado a la práctica.
- Número de personas encargadas. Un municipio pequeño necesitará una persona o dos como mucho. Un número elevado de encargados suele derivar en descontrol y desorganización, pues equivaldría a no tener esa tarea asignada dada la poca importancia que le suponen. Una buena organización de personal y horario debería derivar en una buena comunicación.

- Lanzamiento de campañas publicitarias. No es imprescindible apoyarse en publicidad para promocionarse, pero sí lo es la diferenciación e identificación de las campañas institucionales. Por ejemplo, un evento para generar publicity o una continua presencia en redes sociales, enviando artículos y reportajes a páginas de turismo rural, prensa y blogs especializados, etc.

- Herramientas comunicativas internas. Usadas para informar a los propios habitantes $y$ al personal del Ayuntamiento. Debería ser ésta la prioridad comunicativa municipal, dependiendo de ello conseguir una buena actitud de la plantilla de la corporación y la ciudadanía hacia la comunicación. La cultura de la participación es fundamental en la época actual.

- Herramientas comunicativas externas. Utilizadas en la promoción cultural, turística o económica del municipio, con el fin de atraer habitantes y visitantes. De su eficacia dependerá la notoriedad del ayuntamiento y el retorno gracias a esos intangibles. 
- Definición explícita de valores diferenciales. Aquellos que el propio ayuntamiento se atribuye. Pueden ser materiales (un castillo medieval), de ambiente (cercanía, tradición naturaleza, tranquilidad), subjetivos (paisajes, gente amable) o sociales (eventos o actividades que se ofertan). Es clave tener claro qué destacar para una buena comunicación.

- Eventos importantes que se realizan anualmente. El valor de esta información radica esencialmente en la posibilidad de investigar la presencia de estos eventos en los medios de comunicación, como noticia o anuncio, o en los medios propios.

No profundizar en exceso en ninguno de los parámetros permitirá una comparación fácilmente abarcable de los mismos, una primera aproximación general, tal y como se ha especificado en el propósito de esta investigación. El interés de los datos recabados con esta herramienta radica en establecer una posterior correspondencia entre el tamaño y los recursos de cada localidad con la disposición de su departamento de comunicación y la inversión realizada en este concepto. Además, profundizando en las herramientas utilizadas por los municipios, puede medirse la funcionalidad que tenga la comunicación, por ejemplo, en su presencia en medios, la notoriedad entre la población de la provincia o la actitud que muestre la ciudadanía local hacia la comunicación de su ayuntamiento.

\subsubsection{Obtención de datos para la investigación}

A la hora de obtener los resultados se ha realizado un contacto directo con cada ayuntamiento con el fin de recabar determinado tipo de información cualitativa, que podrá ser empleada para valorar con mayor certeza cada caso. Es importante obtener esa información de forma directa, a pesar de que existan vías más sencillas, ya que el propio proceso de análisis es, en sí, una acción de comunicación que puede sugerir nuevas variables a tener en cuenta o datos de interés.

La vía seleccionada ha sido la entrevista telefónica, pues el e-mail tarda en ser respondido y no permite una comunicación verbal para ajustar y precisar el sentido de la investigación. Por otro lado, concertar entrevistas presenciales supone una preparación previa y hace mucho más complejo el proceso. Llamando puede obtenerse información tan relevante como la existencia en centralita de una extensión de prensa, la identificación de la persona responsable de comunicación por parte de quien atiende. También se puede valorar si esa misma persona puede responder las preguntas, demostrando una implicación real en la valoración de esta demanda informativa. Este tipo de cuestiones ponen de manifiesto la organización comunicativa y, por tanto, la capacidad de comunicar bien. La vía de acceso a la información a través de redes, de forma directa, se podría valorar para corporaciones municipales de mayor dimensión. Para completar o contrastar la información obtenida de las entrevistas telefónicas, puede buscarse información en sus webs o usarse herramientas oficiales, lo que representa la vertiente documental de la investigación. Se proponen:

- El Instituto de Estadística y Cartografía de Andalucía1. Se trata de una fuente oficial en la que hay que tener en cuenta la actualización 
de datos, pero que sirve para aproximaciones y comparativas a una misma fecha.

- "Gobierto", web creada como iniciativa privada que ofrece herramientas de consulta e información a los municipios, donde se vuelcan los presupuestos de los ayuntamientos españoles $^{2}$ Se trata de una herramienta de transparencia que es muy útil en el análisis propuesto.

- El buscador de Renta Per Cápita por municipios que facilita el diario El País, conectado directamente con los datos del Ministerio de Hacienda y más sencillo de utilizar ${ }^{3}$.

\subsubsection{Trabajar con los datos obtenidos}

Es importante organizar los datos en tablas para poder cruzar comparativamente los parámetros, teniendo en cuenta que se trata, en cualquier caso, de una primera aproximación y de un diagnóstico inicial. Los municipios pueden ordenarse en cuanto a población, tamaño, renta per cápita, presupuesto total y porcentaje que representa el presupuesto de comunicación con respecto al total presupuestado en el ejercicio anual. Pueden clasificarse también según tengan o no gabinete de comunicación, trabajen o no periodistas en él, además de tener en cuenta la presencia de un horario definido y el número de personas que conforma este departamento o su sustituto práctico. Cabe pensar que, a mayor volumen de población, tamaño, etc., mayor será la apuesta por la comunicación, aunque no siempre son variables con una relación directamente proporcional.

Por ello, se ha considerado importante estudiar la correlación entre la capacidad socioeconómica de la localidad y los recursos invertidos en comunicar o la presencia de gabinete bien estructurado, es decir, si aquella localidad que más posibilidades económicas y necesidad tiene, por tamaño y población, es aquella que más invierte en comunicación. También es interesante determinar qué ayuntamientos realizan un mayor esfuerzo relativo en función de sus posibilidades. Aunque en valores absolutos la inversión en comunicación sea menor, los indicadores proporcionales ofrecerán una interesante información en torno a la política de comunicación de cada gobierno municipal.

Por otro lado, puede realizarse un análisis valorativo de la comunicación en aspectos como la existencia de una web útil y estructurada, el lanzamiento de campañas que pongan en relieve sus valores diferenciales y sus eventos más importantes 0 , especialmente, si se generan y difunden noticias en los medios de comunicación. Esto último, un estudio de impacto informativo, sería un factor externo, complementario e a esta herramienta e integrable en la misma. También se ha considerado relevante realizar una valoración de la eficacia comunicativa interna y externa, estableciendo un mapa de posicionamiento en notoriedad y actitud. En estos casos se pondrá de manifiesto la utilidad de las herramientas utilizadas por los municipios, que pueden representar una referencia de buenas prácticas para instituciones de características similares. 
Figura 1. Resumen de la herramienta de análisis propuesta

\section{Comunicación corporativa local} Modelo de análisis

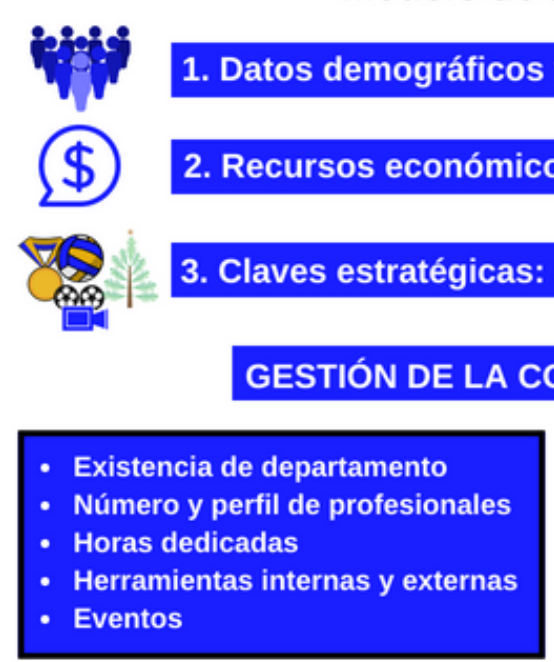

(Mesa-Medina y Marfil-Carmona, 2018)

Fuente: elaboración propia en canva.com

4.2. Puesta en prueba del modelo en $\mathbf{5}$ con el fin de, o bien recabar los datos necesarios, o

\section{municipios}

Como validación y primer ensayo analítico del modelo, se han aplicado estas variables a los cinco ayuntamientos del Área Metropolitana de Granada (España) - Albolote, Alfacar, Cúllar Vega, Maracena y Monachil -, con resultados positivos, en líneas generales, desde el punto de vista profesional de la comunicación institucional. Se divide la puesta en prueba en varias fases, organizando y exponiendo de forma esquemática la aplicación del modelo de análisis.

\subsubsection{Fase 1. Obtención de datos para la} investigación

En esta fase, se han usado las herramientas propuestas para la obtención de datos a través de llamadas telefónicas a los diferentes consistorios bien concertar una posterior entrevista al efecto. Las preguntas que se han realizado son las siguientes:

- ¿Su ayuntamiento cuenta con un departamento dedicado solo a comunicación?

- De no contar con este departamento, ¿quién asume esas tareas?

- ¿Cuántas personas conforman ese departamento?

- ¿Las personas encargadas de ese departamento son periodistas?

- ¿Qué herramientas usan para comunicarse con su población? 
- ¿Qué herramientas usan para promocionarse hacia el exterior?

- ¿Qué aspecto destacaría de su localidad para lanzar un anuncio?

- ¿Lanzan campañas publicitarias en los medios de comunicación?

- ¿Qué eventos importantes genera anualmente?

- ¿Qué presupuesto anual maneja el ayuntamiento?

- ¿Qué parte del presupuesto anual dedica a comunicación?

Debido a la falta de precisión en los datos económicos y sociodemográficos ofrecidos se ha acudido a "Gobierto", buscador de Renta Per Cápita por municipios de El País y al Instituto de Estadística y Cartografía de Andalucía. Era algo esperado, aunque se quería comprobar la capacidad de respuesta al pedir dicha información. Solo se ha tenido acceso a la partida oficial destinada a comunicación del presupuesto anual de Maracena, de modo que en el resto de los casos los datos no han podido ser contrastados o bien son aproximados. Destacan, por tanto, algunos resultados procedimentales de estas llamadas. No existe extensión de prensa en la centralita, tampoco en aquellos ayuntamientos que cuentan con un departamento de comunicación.

Los ayuntamientos sin gabinete o departamento de comunicación no tienen claro quién debe dar esa información y la persona elegida no siempre tiene el conocimiento requerido. Cuando atiende un periodista, facilita mejor algunos datos como el valor diferencial, por estar familiarizado con la gestión de información. Contar con un departamento de prensa permite que otro departamento no pierda su tiempo en responder, tanto en esta recogida de datos como para cualquier consulta semejante, una tarea ajena y que no siempre van a saber realizar.

En la Tabla 1 pueden observarse los datos económicos y sociodemográficos obtenidos; en la Tabla 2, se encuentran los datos relativos al gabinete de prensa; en la Tabla 3, los datos relativos a la comunicación que se lleva a cabo. La presencia de datos poco concretos se corresponde con las respuestas que han dado los propios ayuntamientos.

Tabla 1. Datos demográficos y económicos

\begin{tabular}{|c|c|c|c|c|c|}
\hline Municipio & Población & $\begin{array}{c}\text { Tamaño } \\
\left(\mathrm{en} \mathrm{Km}^{2}\right)\end{array}$ & $\begin{array}{c}\text { Renta per } \\
\text { cápita } \\
\text { (Miles } € \text { ) }\end{array}$ & $\begin{array}{c}\text { Presupuesto } \\
\text { anual (Millones } \\
€ \text { ) }\end{array}$ & $\begin{array}{c}\text { Partida para comu- } \\
\text { nicar }\end{array}$ \\
\hline Albolote & 18.620 & 79 & 27.977 & 14,4 & Unos 15.000 $€$ \\
\hline Alfacar & 5.490 & 16,73 & 19.964 & 4,16 & No existe \\
\hline Cúllar Vega & 7.198 & 4,35 & 21.090 & 4,4 & $\begin{array}{c}7.000 € \text { y un salario } \\
\text { de 1200 } €\end{array}$ \\
\hline Maracena & 22.059 & 4,89 & 19.706 & 17,8 & $\begin{array}{c}\text { Sin cantidad } \\
\text { establecida, varía } \\
\text { según las } \\
\text { necesidades. }\end{array}$ \\
\hline Monachil & 7.681 & 88,92 & 22.615 & 9 & $6.000 €$ \\
\hline
\end{tabular}

Fuente: elaboración propia, basada en las webs oficiales. 
Tabla 2. Datos sobre el gabinete de prensa.

\begin{tabular}{|c|c|c|c|c|c|}
\hline Municipio & $\begin{array}{c}\text { ¿Tiene } \\
\text { gabinete de } \\
\text { prensa? }\end{array}$ & $\begin{array}{c}\text { ¿Quién se } \\
\text { encarga si no } \\
\text { de informar? }\end{array}$ & $\begin{array}{c}\text { ¿Existe } \\
\text { horario } \\
\text { fijo? }\end{array}$ & $\begin{array}{c}\text { ¿El } \\
\text { responsable } \\
\text { es periodista? }\end{array}$ & $\begin{array}{c}\text { Trabajadores } \\
\text { encargados }\end{array}$ \\
\hline Albolote & Sí & - & Sí & Sí & 1 persona \\
\hline Alfacar & No & $\begin{array}{c}\text { No existe esa } \\
\text { figura }\end{array}$ & No & No & $\begin{array}{c}2-3 \text { personas le } \\
\text { dedican tiempo }\end{array}$ \\
\hline Cúllar Vega & Sí & - & No & Sí & 1 persona externa \\
\hline Maracena & Sí & - & Sí & Sí & $\begin{array}{c}1 \text { persona con } \\
\text { equipo externo }\end{array}$ \\
\hline Monachil & No & $\begin{array}{c}\text { Concejal de } \\
\text { comunicación }\end{array}$ & No & No & $\begin{array}{c}1 \text { persona con } \\
\text { apoyo externo }\end{array}$ \\
\hline
\end{tabular}

Fuente: elaboración propia mediante entrevistas telefónicas a los ayuntamientos.

Tabla 3. Datos sobre la comunicación

\begin{tabular}{|l|l|l|l|l|l|}
\hline Municipio & \multicolumn{1}{|c|}{$\begin{array}{c}\text { ¿Lanza } \\
\text { publicidad? }\end{array}$} & $\begin{array}{l}\text { Herramientas } \\
\text { internas }\end{array}$ & $\begin{array}{l}\text { Herramientas } \\
\text { externas }\end{array}$ & $\begin{array}{c}\text { Valor } \\
\text { diferencial }\end{array}$ & \multicolumn{1}{|c|}{ Eventos } \\
\hline Albolote & Sí & $\begin{array}{l}\text { RR.SS. y web } \\
\text { App. móvil } \\
\text { Tablón virtual } \\
\text { Cartelería }\end{array}$ & $\begin{array}{l}\text { Contacto con } \\
\text { medios }\end{array}$ & $\begin{array}{l}\text { Historia } \\
\text { Naturaleza }\end{array}$ & $\begin{array}{l}\text { Candelaria } \\
\text { Romería }\end{array}$ \\
\hline Alfacar & Sí & $\begin{array}{l}\text { RR.SS. } \\
\text { Tablón virtual }\end{array}$ & Ninguna & Industria & $\begin{array}{l}\text { Fiestas } \\
\text { patronales }\end{array}$ \\
\hline Cúllar Vega & $\begin{array}{l}\text { No les inte- } \\
\text { resa }\end{array}$ & $\begin{array}{l}\text { Voto online } \\
\text { RR.SS. y web } \\
\text { App. móvil }\end{array}$ & $\begin{array}{l}\text { Notas de } \\
\text { prensa } \\
\text { RR.SS. y web }\end{array}$ & $\begin{array}{l}\text { Tradición } \\
\text { Implicación }\end{array}$ & $\begin{array}{l}\text { Fiestas } \\
\text { patronales } \\
\text { Día de la Cruz } \\
\text { Secaderos }\end{array}$ \\
\hline Maracena & Sí & $\begin{array}{l}\text { RR.SS. y web } \\
\text { Correo } \\
\text { Cartelería } \\
\text { Medios locales }\end{array}$ & $\begin{array}{l}\text { Medios locales } \\
\text { Convenios con } \\
\text { medios }\end{array}$ & $\begin{array}{l}\text { Referente } \\
\text { metropolitano } \\
\text { Servicios }\end{array}$ & $\begin{array}{l}\text { Gala deportiva } \\
\text { San Antón } \\
\text { Mercado } \\
\text { medieval }\end{array}$ \\
\hline Monachil & Sí & $\begin{array}{l}\text { App móvil } \\
\text { RR.SS. y web }\end{array}$ & $\begin{array}{l}\text { Notas de } \\
\text { prensa } \\
\text { Inserciones }\end{array}$ & $\begin{array}{l}\text { Naturaleza } \\
\text { Sierra Nevada }\end{array}$ & $\begin{array}{l}\text { San Antón } \\
\text { Copa Covap } \\
\text { Mercado de arte }\end{array}$ \\
\hline
\end{tabular}

Fuente: elaboración propia mediante entrevistas telefónicas a los ayuntamientos.

\subsubsection{Fase 2. Organización de los datos}

Si bien una muestra de cinco municipios es pequeña para obtener conclusiones relevantes en lo que atañe a un análisis comparativo, los resultados concretos permiten una interpretación de tendencia o de los aspectos comunes de estos casos concretos.

\subsubsection{Presencia de gabinetes y capacidad económica de los municipios}


De los datos obtenidos en la Tabla 1, se puede de prensa y qué porcentaje del total de la muestra extraer qué número de municipios (3) tienen gabinete representan (un 60\%). Se muestra en la Figura 2.

Figura 2. Presencia de gabinete de prensa

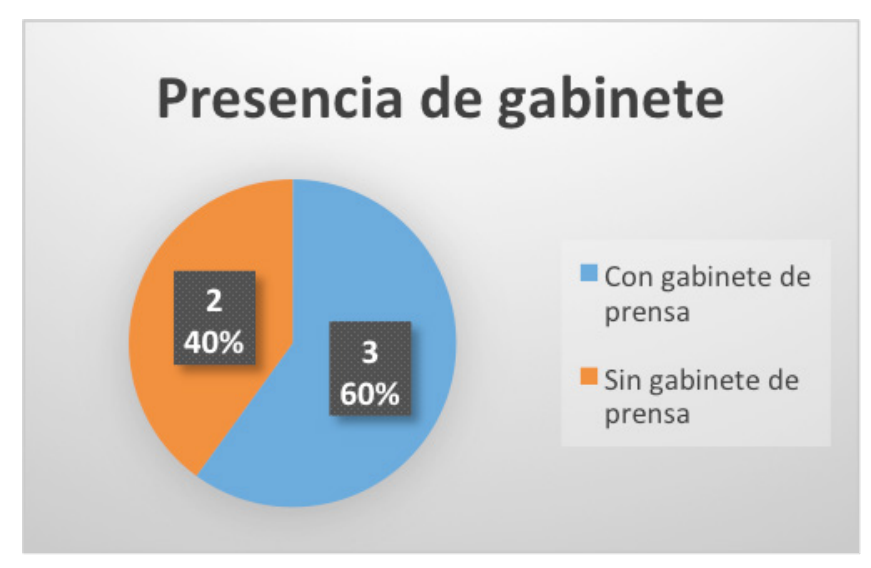

Fuente: elaboración propia.

También puede estudiarse la presencia de partidas tiene una cantidad definida, y Monachil, a pesar de no presupuestarias que, en este caso, no coincide con la contar con gabinete, sí que tiene fijada una partida. presencia de gabinetes: Maracena, con gabinete, no Se muestra en la Figura 3.

Figura 3. Presupuesto de comunicación

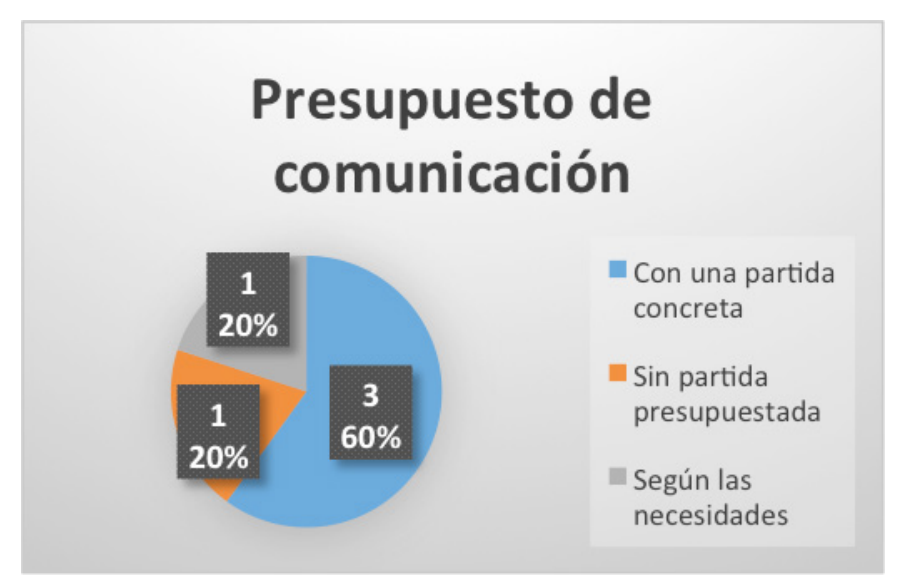

Fuente: elaboración propia.

Por otro lado, quizá una de las claves sea comprobar si los ayuntamientos con mayor número de habitantes (directamente ligado al presupuesto anual según se muestra en la Tabla 4) son aquellos que invierten una mayor cantidad de dinero en comunicar. Resulta interesante ordenar los ayuntamientos por cada variable socioeconómica para comprender su situación. Así, se comprueba que la Renta Per Cápita o el tamaño apenas inciden en el presupuesto, como sí lo hace la población. 
Tabla 4. Ayuntamientos ordenados

\begin{tabular}{|l|l|l|l|l|}
\hline PUESTO & POBLACION & $\begin{array}{l}\text { Renta Per } \\
\text { Cápita }\end{array}$ & PRESUPUESTO & TAMAÑO \\
\hline $\mathbf{1}$ & Maracena & Albolote & Maracena & Monachil \\
\hline $\mathbf{2}$ & Albolote & Monachil & Albolote & Albolote \\
\hline $\mathbf{3}$ & Monachil & $\begin{array}{l}\text { Cúllar } \\
\text { Vega }\end{array}$ & Monachil & Alfacar \\
\hline $\mathbf{4}$ & Cúllar Vega & Alfacar & Cúllar Vega & Maracena \\
\hline $\mathbf{5}$ & Alfacar & Maracena & Alfacar & $\begin{array}{l}\text { Cúllar } \\
\text { Vega }\end{array}$ \\
\hline
\end{tabular}

Fuente: "Gobierto", webs oficiales y Ministerio de Hacienda y Función Pública.

4.2.2.2 Estructura de los gabinetes y esfuerzo económico relativo de los municipios

Los datos presentes en la Tabla 2 permiten profundizar algo más. Ya se ha comprobado que 3 de los 5 municipios cuentan con gabinete de prensa: Maracena, Albolote y Cúllar Vega. Por su parte, Alfacar (5.400 habitantes y 4,4 millones de presupuesto) y Monachil (7.600 habitantes y 9 millones de presupuesto) no creen necesitarlo, aunque Monachil tiene una Concejalía de Comunicación que incluye estas funciones. En todos los casos, la presencia de gabinete implica la contratación de una persona titulada en Periodismo al cargo y la ausencia de gabinete no es suplida con un periodista. Este sería otro aspecto interesante para analizar en lo que respecta al perfil de los profesionales.

Puede ser relevante estudiar la organización de la comunicación. En este caso, salvo Alfacar (3 personas), todos tienen una sola persona al cargo y tan solo Albolote y Maracena establecen un horario para el encargado.

Figura 4. Número de personas que trabajan la comunicación en los municipios

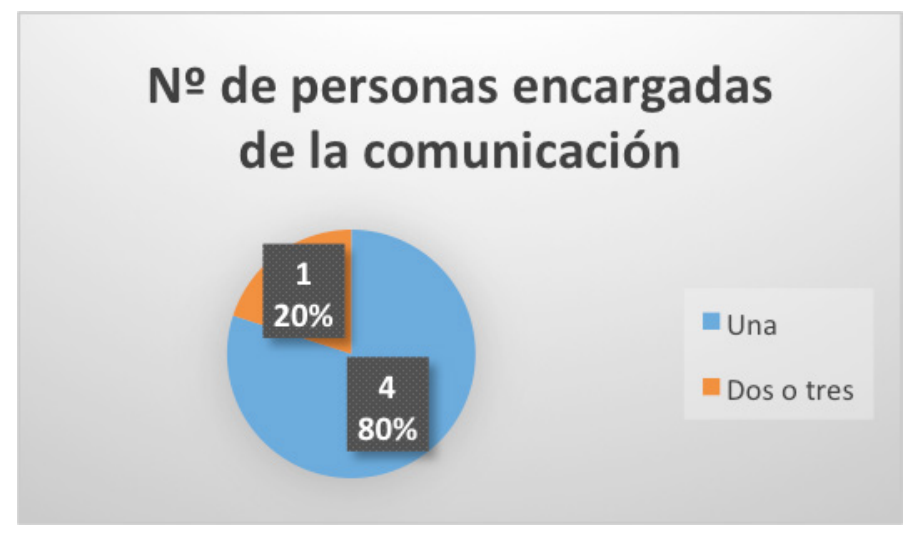

Fuente: entrevistas con los ayuntamientos.

Ala hora de comprobar el criterio de los ayuntamientos la partida destinada a comunicación representa en es importante no trabajar con cantidades totales de inversión, si no relativas al presupuesto que manejan. En la Tabla 5, se muestra el porcentaje que el presupuesto anual. Las cantidades que aparecen como Inversión en comunicación se han estimado preguntando a los responsables. 
Tabla 5. Presupuesto de comunicación de los municipios

\begin{tabular}{|c|c|c|c|}
\hline Municipio & $\begin{array}{c}\text { Presupuesto } \\
\text { anual } \\
\text { (en millones de } \\
\text { euros) }\end{array}$ & $\begin{array}{c}\text { Inversión en } \\
\text { comunicación }\end{array}$ & $\begin{array}{c}\text { Inversión } \\
\text { relativa }\end{array}$ \\
\hline Albolote & 14,4 & $20.000 €$ & $0,14 \%$ \\
\hline Alfacar & 4,16 & $1.500 €$ & $0,03 \%$ \\
\hline $\begin{array}{c}\text { Cúllar } \\
\text { Vega }\end{array}$ & 4,4 & $19.000 €$ & $0,43 \%$ \\
\hline Maracena & 17,8 & $18.000 €$ & $0,10 \%$ \\
\hline Monachil & 9 & $6.000 €$ & $0,07 \%$ \\
\hline
\end{tabular}

Fuente: entrevistas telefónicas con los ayuntamientos.

Realizando este análisis pueden obtenerse varios datos de interés:

- ¿Los ayuntamientos con mayor presupuesto destinan cantidades mayores a comunicar? En esta pequeña muestra, ya observamos casos como Monachil, que apenas invierte 6.000€, o Cúllar Vega, que compite con los grandes consistorios incluso en términos absolutos de inversión.

- ¿Los porcentajes relativos a la inversión comunicativa son semejantes? En estos municipios no lo son en absoluto, representando un $0^{\prime} 43 \%$ en Cúllar Vega mientras que Alfacar o Monachil apenas llegan al $0,1 \%$.

- ¿Qué cantidad media se destina a comunicar? En este caso, 12.900€. Se trata de un dato curioso en lo relativo a la gestión. Hay que detraer, por supuesto, los gastos porcentuales de equipamientos e infraestructuras puestas a disposición de la acción comunicacional.

4.2.2.3 . Eficacia publicitaria e idoneidad de las herramientas
Trabajando con los datos que se muestran en la Tabla 3 , puede estudiarse si los municipios que cuentan con gabinete de comunicación son aquellos que lanzan campañas publicitarias. En este caso, tanto Alfacar como Monachil contrata publicidad sin contar con gabinete, mientras que Cúllar Vega no lo considera necesario. En un dato bastante subjetivo como el 'valor diferencial' del municipio, Alfacar ha mostrado carencias en el concepto y Monachil ha respondido quizá generalidades. No es fácil sintetizar las claves fundamentales de la línea de comunicación. Se reconoce, en este sentido, que la presencia de periodistas resulta positiva en líneas generales, aportando para definir las propias señas de identidad, el contenido a difundir. Los casos estudiados presentan algunos aspectos comunes en lo que es una progresiva apuesta por la comunicación en las corporaciones municipales.

\section{CONCLUSIONES Y DISCUSIÓN}

\subsection{Conclusiones}

Se ha considerado relevante diseñar una herramienta para obtener los datos precisos que se requieren para estudiar la comunicación de los municipios pequeños, 
abarcando la mayor parte de aspectos que determinan el modelo de comunicación de los ayuntamientos estudiados. Tal y como se sintetizaba en los objetivos principales de la investigación, la herramienta ha permitido analizar:

- La capacidad económica del municipio y su inversión en comunicación mediante datos cuantitativos y aspectos sociodemográficos. De los datos relativos que se han analizado puede obtenerse el criterio presupuestario del ayuntamiento en materia comunicacional. Disponer de más dinero no quiere decir que se invierta más en comunicación.

- La existencia de gabinete de prensa, su composición y su organización interna, constatando que no hay un criterio común en las localidades analizadas.

- El lanzamiento de campañas publicitarias y las herramientas que usan estas localidades para comunicase con públicos internos y externos, así como la posibilidad de medir su eficacia. Solo una de las cinco localidades analizadas no apuesta por contratación de publicidad, justo la que tiene una mayor inversión relativa en comunicación.

- El posicionamiento, como valor diferencial, que creen tener en sus municipios y los eventos más importantes que promocionan a lo largo del año. Esto brinda la posibilidad de analizar, posteriormente, su presencia en los medios de comunicación. En determinadas localidades, no se tienen demasiado definidos los atributos de identidad y las líneas principales de contenidos a difundir.

No tendría sentido aplicar estas variables en una ciudad de mayores dimensiones, pues los ayuntamientos de gran tamaño tienen un gabinete de prensa profesionalizado que, normalmente, está coordinado por un cargo de confianza del grupo político que gobierna en ese momento. En los consistorios más pequeños sí que tiene sentido implementar la herramienta, lo que constituye la principal conclusión del reto metodológico que conforma, en sí misma, esta investigación.

Es importante destacar la relevancia de la obtención de datos en sí. No se trata solo de obtener una serie de ítems, las vías son importantes. En los apartados anteriores se justificaba la necesidad de comunicarse directamente con el propio ayuntamiento a la hora de recabar sensaciones subjetivas y conocer de primera mano la organización interna en el apartado comunicativo para formarse una imagen del mismo. La valoración de ese procedimiento es altamente positiva, pues supone un proceso de aportación continua y aprendizaje.

Con los datos recabados han podido analizarse los municipios y sus gabinetes de prensa desde bastantes puntos de vista diferentes, tanto cuantitativa como cualitativamente, resultando posible profundizar en una o varias de estas cuestiones, o bien abarcarlas todas ofreciendo una visión mucho más amplia. Las opciones ofrecidas en la puesta en prueba que se ha llevado a cabo en este trabajo son tan solo parte de las posibilidades analíticas que permiten los datos recabados mediante esta herramienta. El propio investigador debe decidir qué puntos de vista requiere su proyecto. 
La herramienta, por tanto, ha resultado útil a la hora de analizar en un espacio breve de tiempo el interés y la capacidad de comunicar de los cinco municipios. Se trata de una muestra muy reducida para llegar a conclusiones extrapolables a un área geográfica, pero suficiente para validar la eficacia de la metodología empleada. Sí que se ha detectado, en la muestra, poca adaptación a las nuevas formas de comunicación si las comparamos con el mundo empresarial, una sugerencia para futuras investigaciones que se une a las líneas aportadas la discusión.

\subsection{Discusión}

Esta investigación constituye una aportación de utilidad para la aplicación al sector de las corporaciones locales. Así, con respecto a la ruptura del paradigma actual en la comunicación política (Rodríguez-Andrés, 2016) que debe tener muy presente el nuevo contexto digital (Almansa Martínez, 2008; Campillo Alhama y Ramos-Soler, 2013), los resultados de la aplicación de la herramienta diseñada muestran claramente cómo el proceso de adaptación es desigual en cada ayuntamiento, siendo imprescindible la valoración de cada aspecto que influya en la eficacia comunicacional. De igual forma, la valoración de los datos contextuales donde las instituciones desarrollan su actividad representa una aproximación analítica de gran interés, necesaria para la estrategia, en los parámetros clave comentados en la revisión teórica de este trabajo y aportados por Domínguez (2009), ya que la notoriedad es, en su efecto más inmediato, la capacidad de influir en la propia opinión pública, siendo también un indicador de la actitud de una corporación con respecto al reto de comunicar con eficacia, aspecto en el que inciden Castillo-Esparcia y Smolak-Lozano (2016, p. 329). Además, esa tarea es útil, sin duda, para vertebrar el territorio, tal y como afirma Lluís Costa (2015). La base de conexión y relación con la ciudadanía es fundamental, especialmente en el nuevo modelo comunicacional al que se ha hecho referencia (Marta-Lazo y José Antonio Gabelas, 2016; Pulido Polo, 2013).

Esa eficacia no es directamente proporcional a disponer de más dinero, según la puesta en prueba realizada. Por ello, a la necesidad de contar con un presupuesto, recogida por Joan Costa (2014), hay que unir inevitablemente el criterio profesional y la capacidad estratégica, en la línea de profesionalización sugerida por Almansa (2004). Como se comprueba en los resultados del estudio en 5 localidades, se puede dar el caso de invertir el presupuesto en comunicación en mayor medida que en publicidad. Lo limitado de la muestra nos lleva a ser prudentes en torno a la garantía de éxito al aplicar este criterio, lo que hace recomendable la ampliación del estudio para obtener una perspectiva más detallada y aplicable al contexto de la comunicación local. El problema de partida, en este sentido, sería la consideración del trabajo para generar publicity, frente a las clásicas campañas publicitarias.

En lo que respecta a la claridad en el criterio, comprobamos que no todos los casos evidencian tener claros sus atributos, sus señas de identidad como municipios, tal y como sí ocurre en algunas grandes ciudades comentadas (Álvarez, 1999; Cócola, 2010; Sáez, 2011), por lo que hay que insistir en que se trata de una acción clave, absolutamente recomendable. Un primer paso es la valoración de ese "patrimonio intangible", el contenido para posicionarse al que hacían referencia Jiménez y San Eugenio (2009, pp. 266-287). Esa clarificación, que es recomendable 
realizar desde la visión profesional de la comunicación, aunque en ese proceso de especialización profesional recomendado por las investigaciones del sector (Armendáriz, 2015; Capriotti, 2016; Escobar y Gómez, 2015; Martín, 2007; Subiela Hernández, 2013; Villafañe, 2011) no hay un criterio común por parte de las corporaciones locales estudiadas, lo que permite afirmar que existe una disparidad entre ellas y que, en el propio sector, no es arriesgado aventurar que estas decisiones se ven influidas por el propio contexto humano y de gestión. En este análisis, además, se constata que se trata de un tipo de corporaciones con unas características muy especiales, en comparación con las grandes instituciones públicas. Las investigaciones reseñadas, como la Vázquez Gestal y Fernández Souto (2014), advertían sobre los rasgos específicos del sector local. La selección de esos contenidos a difundir es una primera vía para seleccionar entre miles de datos posibles, es decir, en un contexto de verdadera infoxicación en la comunicación corporativa, al que hacen referencia Romero-Rodríguez y Mancinas-Chávez (2018, p. 117). Esta investigación, en resumen, constata algunas líneas de investigación reseñadas, en una línea que puede continuar mediante la aplicación de la herramienta de análisis diseñada.

Con respecto al proceso de investigación en sí, surgen varias cuestiones en las que merece la pena detenerse. En primer lugar, se ha planteado hacer partícipe al objeto de estudio en la recolección de datos, lo que conlleva un sistema de transferencia y retorno del esfuerzo investigador a la institución. Como aspecto positivo, contar con información obtenida directamente de las personas que trabajan en esas corporaciones genera un feedback durante el proceso que aporta calidad al análisis y, sobre todo, a la hora de entender y contextualizar ciertos resultados.

Sin embargo, este factor puede hacer que la información sea más inestable y subjetiva, pues no todas las personas con las que se ha contactado estaban capacitadas para responder a las preguntas de la mejor manera posible. Se ha recabado mucha información irrelevante que se ha tenido que desechar, y quizá sea éste un buen punto de partida para comenzar un proyecto de investigación: no todos los ayuntamientos contaban, no ya con gabinete de prensa, si no con alguien que supiera responder a las cuestiones, con una persona que se manejara en el terreno. Una clara evidencia de las necesidades de comunicación en este tipo de corporaciones.

En los casos estudiados, se ha respondido bien al contacto, pero sin duda puede haber un número elevado de ayuntamientos con los que sea imposible contactar por diversos motivos. Quizá sea más productivo personarse para entrevistar al objeto de estudio a fin de, no solo "provocar" una respuesta efectiva, sino también sacar muchísimo más provecho a los intangibles que se mencionaban: actitud, interés, importancia que se da a las herramientas o los conceptos que se manejan... En definitiva, trabajar cara a cara para enriquecer la obtención de datos.

No se trata de un modelo que permita analizar en profundidad un aspecto comunicativo aislado. Su objetivo es otro: ofrecer una visión global sobre el criterio de gestión de la comunicación de los municipios, tocando la mayor parte de aspectos que la componen. El contraste de otras variables constituye una línea de investigación en el futuro.

Este estudio, por último, tiene un claro carácter prospectivo. Es una herramienta y carecerá de utilidad 
si no se le da uso. El siguiente paso podría ser ampliar la muestra a todo el Área Metropolitana de Granada, o a toda una provincia, por ejemplo, despejando definitivamente las dudas sobre su utilidad al analizar este objeto de estudio en todas sus dimensiones. Puede servir asimismo como modelo de análisis para otro tipo de instituciones locales, diputaciones provinciales o gobiernos autónomos. El sector público tiene mucho camino por desarrollar para garantizar una mayor transparencia y una mejor comunicación con la ciudadanía.

\section{NOTAS}

${ }^{1}$ Página web del Instituto de Estadística y Cartografía de Andalucía disponible en https://goo.gl/DRvuWS (Fecha de consulta: 1/03/2018).

2 Página web de "Gobierto" disponible en https://presupuestos.gobierto.es/ (Fecha de consulta: 27/02/2018).

${ }^{3}$ Buscador de renta media de El País, con las 2964 localidades de España con más de 1.000 habitantes. Disponible en https://goo.gl/CCe6F7 (Fecha de consulta: 26/02/2018).

\section{REFERENCIAS}

Aced, C. (2013). Relaciones públicas 2.0: Cómo gestionar la comunicación corporativa en el entorno digital. Barcelona: UOC.

Almansa Martínez, A. (2004). Historia de los gabinetes de comunicación en España. Historia y comunicación social, 9 , 5-21. Recuperado de https://goo.gl/XAY7WK

Almansa Martínez, A. (2008). Estudio sobre el uso de las nuevas tecnologías en comunicación institucional. Icono 14, (11), 1-14. Recuperado de https://goo.gl/Ldh4q4

Álvarez Mora, A. (1999). Bilbao, la definición de una 'imagen de marca' como reclamo competitivo. Valladolid: Universidad de Valladolid.

Armendáriz, E. (2015). El nuevo perfil del profesional de la Comunicación y las Relaciones Públicas. Una visión desde la perspectiva del mercado. Revista Internacional de Relaciones Públicas, 5(9), 153-178. http://dx.doi.org/10.5783/ RIRP-9-2015-09-153-178

Campillo Alhama, C. y Ramos-Soler, I. (2013). La comunicación 2.0 de las políticas orientadas a mayores en los ayuntamientos españoles. Estudios sobre el mensaje periodístico, 19, 661-670. https://doi.org/10.5209/rev_ ESMP.2013.v19.42149

Capriotti, P. (2016). Branding corporativo. Gestión estratégica de la identidad corporativa. Comunicación, (27), 15-22. Recuperado de https://goo.gl/LzyNa3

Castells, M. (2008). Comunicación, poder y contrapoder en la sociedad red (I). Los medios y la política. Telos, (74). Recuperado de https://goo.gl/DnevFW

Castillo-Esparcia, A. (2010). Introducción a las Relaciones Públicas. Málaga: Instituto de Investigación en Relaciones Públicas. [Versión electrónica]. Recuperado el 24/02/2018 de https://goo.gl/uoqiia

Castillo-Esparcia, A. y Smolak Lozano, E.S. (2016). La comunicación de los think tanks. Análisis de la estrategia digital. Opción, 32(9), 327-342. Recuperado de https://goo.gl/8VLuVG 
Catalina García, B. y García Jiménez, A. (2013). Herramientas interactivas y participación ciudadana en los ayuntamientos españoles. Ámbitos: Revista Internacional de Comunicación, (22). Recuperado de https://goo.gl/tgZgJf

Cócola, A. (2010). El Barrio Gótico de Barcelona. Planificación del pasado e imagen de marca. Barcelona: Universidad de Barcelona.

Costa, J. (2014). El DirCom hoy: dirección y gestión de la comunicación en la nueva economía. Barcelona: Costa Punto Com SL.

Costa, L. (2015). La comunicación local. Barcelona: UOC.

Domínguez, D.C. (2009). Democracia 2.0: La política se introduce en las redes sociales. Pensar la publicidad, 3(2), 31-48.

Escobar, J.M. y Gómez, M. (2015). Diálogos con los DIRCOM (Conceptualizaciones, casos y expertos). Barcelona: Paidós.

García Santamaría, J.V. y Martín Matallana, J. (2017). La transparencia municipal en España: análisis de los factores que más influyen en el grado de transparencia. Revista Latina de Comunicación Social, (72), 1148-1164. https://doi. org/10.4185/RLCS-2017-1212

Igartua, J.J. y Humanes, M.L. (2010). Teoría e investigación en Comunicación Social. Madrid: Síntesis.

Jiménez, M. y San Eugenio, J. D. (2009). Identidad territorial y promoción turística: la organización de eventos como estrategia de creación, consolidación y difusión de la imagen de marca del territorio. Vic (Barcelona): Universidad Central de Catalunya.

Levine F., Locke C., Sears D. y Weinberger d. (2008), El manifiesto Cluetrain. El ocaso de la empresa convencional. Barcelona: Deusto.

Marta-Lazo, C. y Gabelas, J.A. (2016). Comunicación digital. Un modelo basado en el Factor R-elacional. Barcelona: UOC.

Martín, I. (2007). Retos de la comunicación corporativa en la sociedad del conocimiento: de la gestión de información a la creación de conocimiento organizacional. Signo y pensamiento, 26(51), 52-67. Recuperado de https://goo.gl/ vppimw

Muraro, H. (1991). Poder y comunicación: la irrupción del marketing y de la publicidad en la política. Buenos Aires: Ediciones Letra Buena.

Pulido Polo, M. (2013). La perspectiva relacional de las relaciones públicas: una aproximación al valor comunicacional de las indicaciones geográficas. Revista Comunicación, (11), 111-125. Recuperado de https://goo.gl/27exhq

Ramos Ostio, M. J. (2012). Relaciones Públicas 2.0: el uso de los medios sociales en la estrategia de comunicación online de marcas ciudad españolas. Revista Internacional de relaciones públicas, 2(3), 71-90. http://dx.doi.org/10.5783/ RIRP-3-2012-04-71-90

Rodríguez-Andrés, R. (2016). El ascenso de los candidatos outsiders como consecuencia de las nuevas formas de Comunicación Política y la desafección ciudadana. Pozuelo de Alarcón (Madrid): Universidad Francisco de Vitoria.

Romero-Rodríguez, L.M. y Mancinas-Chávez, R. (2016). Sobresaturación informativa: visibilizar el mensaje institucional en tiempos de infoxicación. En L.M. Romero-Rodríguez y R. Mancinas-Chávez (eds.), Comunicación institucional y cambio social (pp. 111-137). Sevilla: Egregius. 
Sáez, L., Mediano, L. y Elizagarate, V. (2011). Creación y desarrollo de marca ciudad. Análisis de los registros de marca de las principales ciudades españolas. Bilbao: Euskal Herriko Universitea.

Scolari, C. (2008). Hipermediaciones: Elementos para una Teoría de la Comunicación Digital Interactiva. Barcelona: Gedisa.

Subiela Hernández, B. (2013). Perfiles profesionales en el área de la Comunicación corporativa. En M.C. Agustín Lacruz, C. Marta-Lazo y M.I. Ubieto Artur (coords.), Perfiles profesionales y espacios de empleo en Información y Comunicación (pp. 109-137). Madrid: Icono 14.

Sureda Pons, M. y Torres Plana, L. (2016). Manual de comunicación para ayuntamientos pequeños (y no tan pequeños). Barcelona: UOC.

Thompson, J.B. (2013). La transformación de la visibilidad. Estudios públicos, (90), 273-296. Recuperado de https://goo. gl/7h95hu

Vázquez Gestal, M. y Fernández Souto, A.B. (2014). El profesional de las Relaciones Públicas en Galicia: el caso de los principales ayuntamientos gallegos. Revista Internacional de Relaciones Públicas, 4(7), 25-38. http://dx.doi. org/10.5783/RIRP-7-2014-03-25-38

Villafañe, J. (2004). La buena reputación: Claves del valor intangible de las empresas. Madrid: Pirámide.

Villafañe, J. (2011). La gestión profesional de la imagen corporativa (4ª ed.). Madrid: Pirámide. 